\title{
Strates
}

STRATES Matériaux pour la recherche en sciences sociales

$12 \mid 2006$

Nouvelles tensions impériales et recompositions en Europe centrale, orientale, et CEI

\section{Comprendre le nouveau régime russe}

Jean-Robert Raviot

\section{(2) OpenEdition}

Journals

Édition électronique

URL : http://journals.openedition.org/strates/1662

DOI : $10.4000 /$ strates. 1662

ISSN : $1777-5442$

Éditeur

Laboratoire Ladyss

Édition imprimée

Date de publication : 31 décembre 2006

ISSN : 0768-8067

Référence électronique

Jean-Robert Raviot, " Comprendre le nouveau régime russe », Strates [En ligne], 12 | 2006, mis en ligne le 19 juillet 2007, consulté le 10 décembre 2020. URL : http://journals.openedition.org/strates/1662 ; DOI : https://doi.org/10.4000/strates. 1662

Ce document a été généré automatiquement le 10 décembre 2020.

Tous droits réservés 


\title{
Comprendre le nouveau régime
}

\section{russe}

\author{
Jean-Robert Raviot
}

1 Le titre choisi ici fait écho à l'ouvrage de référence de Marc Raeff sur l'histoire politique de la Russie, Comprendre l'ancien régime russe ${ }^{1}$. L'auteur de ces lignes n'a pas la longue expérience de l'auteur du livre cité, fruit d'une fréquentation assidue et prolongée des sources et d'une réflexion approfondie portant sur une histoire bien plus longue que cette douzaine d'années d'existence du nouveau régime russe. Cependant, il poursuit l'objectif de replacer la réflexion sur le fonctionnement du régime politique de la Russie contemporaine dans une perspective historique. Le retour à l'histoire et l'attention portée aux continuités sociales, institutionnelles ou culturelles, permet en premier lieu de préciser, sinon de relativiser, voire de démystifier les questionnements qui prévalent aujourd'hui : la Russie est-elle une démocratie? En quoi le régime postsoviétique se démarque-t-il des démocraties occidentales, et pourquoi?

Le règne des poncifs

2 Depuis deux siècles au moins, la Russie occupe une place très particulière parmi les « autres » que l'Occident se construit. Tout à la fois chrétienne et européenne, c'est-àdire civilisée, et «asiatique », c'est-à-dire barbare, la Russie est l'étranger exotique le plus proche de l'Europe, par son histoire et sa géographie. La Russie est un miroir à travers lequel l'Occident perçoit ce qui n'est pas occidental et qui reflète les fantasmes occidentaux sur les traits distinctifs de leur identité. En d'autres termes, l'intérêt des Occidentaux pour la Russie est plus animé par une volonté de se définir que de comprendre la civilisation russe pour ce qu'elle est ${ }^{2}$. La genèse de l'orientalisme au XIX siècle ${ }^{3}$ ainsi que les débats contemporains sur l'appartenance ou non de la Turquie à l'Europe procèdent également de la projection de fantasmes, à la grande différence près que la Russie n'est jamais tout à fait « autre».

$3 \mathrm{Au}$ XIX ${ }^{e}$ siècle, ce sont ces fantasmes et poncifs occidentaux qui définissent l'orientation et la teneur du débat intellectuel en Russie. La haine de soi des intellectuels occidentalistes et le chauvinisme des intellectuels slavophiles sont autant de réponses, bien souvent péremptoires, au même questionnement sommaire : la Russie appartient- 
elle au monde occidental? On voit apparaître en Occident deux versions idéalisées de cet « autre Occident » ou de cet "Orient très proche ». Pour les uns, la Russie est un pays archaïque où règnent la violence et l'arbitraire, la corruption à tous les étages, la vulgarité et la saleté, un pays où la crédulité et l'ignorance font rage. D'autres mettent l'accent sur l'archaïsme russe, qui serait une vertu. En Russie, l'absence de démocratie vaut mieux que la démocratie en trompe l'œil du capitalisme, où la raison et la science n'ont pas encore triomphé de la croyance en Dieu qui protège l'homme du désenchantement.

4 Après la révolution de 1917, ces deux séries de poncifs se perpétuent. Pour les héritiers des russophobes, l'URSS est la patrie de l'oppression aveugle et du totalitarisme. Les sympathisants du socialisme, au sens large du terme, se font plutôt russolâtres : c'est l'heure de la " grande lueur à l'Est ». La guerre froide consolide ce clivage, qui structure la controverse savante entre soviétologues «totalitariens " et «révisionnistes " aux États-Unis. Au-delà de la dimension proprement politicienne d'un débat lié à la définition d'une position américaine dans son rapport avec l'URSS, on constate que bien des «totalitariens" boivent à la source russophobe de la Russie archaïque et despotique par nature et que bien des "révisionnistes" se désaltèrent à celle qui abreuvait, au XIX $x^{e}$ siècle, les thèses du populisme russe qui exaltait les vertus démocratiques d'un peuple qui ployait pourtant sous le joug, l'ignorance et la pauvreté. Cette russolâtrie quelque peu rousseauiste renaît d'ailleurs pendant la Perestroika, où certains ont voulu croire que les réformes gorbatchéviennes traduisaient une demande sociale parvenue jusqu'aux oreilles des dirigeants, signe qu'une "société en mouvement » s'emparait, enfin, des rênes de l'histoire...

5 À l'ère post-soviétique, rares sont ceux qui distinguent à l'Est un signe de renouveau et d'espoir, sinon quelques croisés du marché libre, avant, toutefois, la crise financière d'août 19984. La Russie est devenue, dans la conscience collective occidentale, un « trou noir » géopolitique ${ }^{5}$. Cette expression évoque aussi un pays plongeant dans le chaos, le désordre et la corruption et renouant avec le despotisme, voire la dictature sur fond de déchéance sociale et d'appauvrissement généralisé, sources d'un fascisme rampant dont les manifestations de racisme et d'antisémitisme seraient, en ce début de millénaire, autant d'incontestables signaux... Un regard, même furtif, sur l'image donnée de la Russie d'aujourd'hui dans les médias occidentaux confirme la prégnance de ces poncifs, bien servis par une certaine esthétique de la décadence très prisée par les journaux et magazines. $\mathrm{Si}$, par bonheur, la russolâtrie, qui a culminé avec le mirage soviétique, n'est plus de mise aujourd'hui, on regrette vivement que l'image de la Russie et de ses habitants soit si constamment déformée et caricaturée. La quête, par les Russes, de motifs de fierté nationale est généralement considérée, en Occident, comme vaine, voire pathologiquement « revancharde » ou « impérialiste».

6 Aujourd'hui, le poncif politique le plus répandu est qu'aucune "véritable démocratie » ne peut émerger en Russie. Un autre poncif, moins courant mais néanmoins présent dans le discours savant ${ }^{6}$ ou militant ${ }^{7}$, suggère que l'importation de la " démocratie de marché » occidentale, plus ou moins dictée par la "globalisation néo-libérale », a mis un terme au processus de "réelle démocratisation » amorcé sous Mikhaïl Gorbatchev. Comment, sous le règne des poncifs, comprendre le nouveau régime russe?

Le césarisme originel

7 Le nouveau régime russe est marqué du sceau du césarisme ${ }^{8}$, car il revêt, dès son origine, plusieurs caractéristiques qui s'y rattachent: il s'agit d'un régime démocratique non 
représentatif où le président dispose d'un pouvoir fort dont la légitimité fondatrice réside dans la résolution d'une crise politique. C'est une démocratie née du fiat du premier chef d'État russe de l'histoire élu au suffrage universel direct, Boris Eltsine, à la faveur de la crise qui paralyse le pouvoir au cours des deux premières années de l'existence de la Russie post-soviétique (1992-1993). Le président russe mène par décret une politique de réformes économiques radicales auxquelles le Soviet suprême s'oppose systématiquement. Le conflit entre les pouvoirs exécutif et législatif, qui jouissent tous deux d'une légitimité démocratique récemment acquise, ne trouve pas d'issue. Six mois après un référendum en forme de plébiscite qu'il interprète en sa faveur, Boris Eltsine dissout le Parlement en octobre 1993. Proclamant l'état d'urgence, il fait donner l'assaut contre les élus retranchés à l'intérieur du bâtiment de la Maison blanche, là même où le Président russe avait symboliquement posé sur un char pour les photographes, en août 1991, drapeau tricolore russe à la main, pour défendre les acquis démocratiques contre le putsch anti-Gorbatchev. L'emblématique coup de force du président contre le Soviet suprême constitue l'apogée du césarisme et le point de départ du nouveau régime consacré par la Constitution adoptée le 12 décembre 1993. La primauté présidentielle est désormais constitutionnelle et le caractère présidentialiste du régime ne fait que se renforcer par la suite. Le nouveau régime russe est aujourd'hui souvent qualifié de monarchie électives.

8 La dynamique césariste était en marche depuis 1989. C'était alors que le dernier secrétaire général du PCUS, Mikhail Gorbatchev, lançait sous le mot d'ordre de démocratisation la troisième phase de la Perestroika, qui visait à opérer un transfert du pouvoir du parti, dont le rôle dirigeant est aboli en février 1990, vers l'État. Un an après les premières élections semi-libres de l'histoire de l'URSS, en avril 1989, Mikhaïl Gorbatchev se fait élire président et chef de l'État, une fonction qui, en URSS, était de facto exercée par le secrétaire général du parti. Au sommet de l'État, du parti et à la tête des forcées armées, il cumule tous les pouvoirs régaliens, civils et militaires. Pourtant, son impuissance ne fait que croître. Face à lui, un pôle de pouvoir se constitue autour de Boris Eltsine. Exclu des instances dirigeantes du PCUS en 1987, l'ancien secrétaire du parti de la région de Sverdlovsk, dans l'Oural, puis de la ville de Moscou construit une entreprise politique fondée sur son charisme personnel et un discours réformateur plus radical que celui de Mikhaïl Gorbatchev. Boris Eltsine prône la rupture avec le système soviétique et l'indépendance des républiques qui la souhaitent. Favorable à l'instauration d'une démocratie à l'occidentale et à des réformes économiques libérales, il fait usage d'une rhétorique qui dénonce la corruption et les privilèges de la classe dirigeante, la nomenklatura. Après l'élection de Boris Eltsine à la présidence de la Russie au suffrage universel en juin 1991, la rivalité entre les Présidents russe et soviétique devient l'axe central de la vie politique. Après le putsch manqué contre Mikhaïl Gorbatchev, Boris Eltsine prend les commandes, bénéficiant de l'onction d'un suffrage populaire que M. Gorbatchev, élu Président de l'URSS par le Parlement, n'avait pas eu le courage d'affronter.

9 En octobre 1993, la dynamique césariste donne un coup d'arrêt à l'embryonnaire démocratie représentative russe, en gestation au sein des soviets régionaux et du Soviet suprême, presque tous dissous. Plus qu'un type de régime, le césarisme est en effet une certaine forme d'économie du pouvoir. La notion de césarisme renvoie à des situations historiques très diverses. Outre l'antique césarisme romain, il désigne le Second Empire $^{10}$ (1852-1870), le régime de l'Allemagne du Second Reich (1871-1918) voire ceux, plus contemporains, de la Pologne du maréchal Pilsudski (1926-1935), du Brésil de 
Getulio Vargas ${ }^{11}$ (1937-1944). Bien des régimes, antérieurs comme postérieurs, relèvent du césarisme, en particulier en Amérique latine, terre du caudillisme ${ }^{12}$. Tous ces césarismes ont un point commun : un exécutif fort dominé par un chef politique au leadership charismatique et, en contrepoint, des institutions parlementaires faibles, voire quasi inexistantes. En outre, certains éléments césaristes sont bien présents dans des démocraties représentatives, comme la $\mathrm{V}^{\mathrm{e}}$ République, un régime auquel la Constitution russe post-soviétique se réfère de manière explicite.

La Russie est-elle une démocratie?

Le nouveau régime russe est le fruit d'une dynamique démocratique enclenchée par le haut. La démocratisation gorbatchévienne visait à reconstruire la puissance et à restaurer le crédit international de l'URSS. Après la dislocation de cet État en 1991, le nouveau pouvoir affiche l'objectif de faire de la «nouvelle Russie » une démocratie à l'image des démocraties occidentales à l'appui d'une demande de soutien politique et financier des dirigeants occidentaux. Ainsi, l'option en faveur d'un régime démocratique pluraliste vise-t-elle d'abord à renforcer la légitimité des dirigeants par le biais du suffrage universel plutôt qu'à jeter les bases d'une véritable démocratie libérale.

La métamorphose de la démocratie

11 Avec la fin de la guerre froide et de la confrontation idéologique entre les deux grandes puissances, l'instauration d'une démocratie «de type occidental» est devenue, pour tous les États du globe, un impératif conditionnant leur acceptation au sein de la communauté internationale et un critère déterminant pour l'octroi de certains avantages économiques ou la mise en œuvre de certaines politiques publiques internationales ${ }^{13}$. Aussi tous les États, ou presque, s'efforcent-ils d'apparaître comme démocratiques, en réformant leurs systèmes politiques, voire en se dotant d'institutions nouvelles. Autant dire que les anciennes pratiques n'en disparaissent pas pour autant du jour au lendemain. Ainsi, en raison de son expansion planétaire, la démocratie est-elle devenue protéiforme. La frontière entre démocratie et autoritarisme est devenue très poreuse. À l'échelle de la planète, la plupart des régimes ne se trouvent-ils pas dans ce marais entre démocratie et autoritarisme? En réalité, comme le souligne Fareed Zakaria dans un essai très diffusé à travers le monde ${ }^{14}$, c'est l'essence libérale de la démocratie occidentale qui tend à disparaître au profit d'une nouvelle forme de démocratie qui combine des institutions plus ou moins démocratiquement élues avec un pouvoir oligarchique aux pratiques non démocratiques, voire autoritaires : la démocratie illibérale. Fareed Zakaria désigne sous ce terme deux phénomènes assez différents: la transformation des anciennes démocraties libérales en démocraties de masse, de plus en plus "illibérales »; la transformation des régimes autoritaires en démocraties libérales, souvent de pure façade. Le nouveau régime russe peut être rangé dans cette seconde catégorie. En réalité, les deux phénomènes sont liés car, même s'il est toujours cité en référence, le modèle démocratique libéral n'existe plus guère que dans les manuels de philosophie politique. Ne pouvant donc guère servir de modèle pratique pour les politiques de démocratisation, le modèle réel est celui des démocraties occidentales d'aujourd'hui, des démocraties de masse qui assurent non plus tant la promotion du libéralisme politique que de la gouvernance démocratique. Dans les années 1920, Carl Schmitt écrivait :

Il y a certainement peu d'hommes aujourd'hui qui voudraient renoncer aux vieilles

libertés libérales, à la liberté de parole et de presse. Mais sur le continent européen, 
il n'y en a probablement plus beaucoup qui croient que ces libertés existent encore dès lors qu'elles pourraient devenir réellement dangereuses pour les tenants $d u$ pouvoir réel. Ce qui subsiste sans doute le moins, c'est la croyance que d'articles de journaux, de discussions dans les assemblées et de débats au Parlement émergent la législation et la politique véritables [...] Si l'espace public et la discussion sont devenus une formalité vide et caduque dans la réalité effective de l'activité parlementaire, alors le Parlement aussi, tel qu'il s'est développé au XIX ${ }^{\mathrm{e}}$ siècle, a perdu la base qui le soutenait jusqu'à présent, ainsi que son sens ${ }^{15}$.

$12 \mathrm{Au}$ cours $\mathrm{du} \mathrm{xx}^{\mathrm{e}}$ siècle, le pilier de la démocratie représentative libérale occidentale, l'aristocratie des professionnels de la politique ${ }^{16}$, organisée en partis institutionnalisés porteurs des intérêts des différentes classes sociales et d'idéologies politiques correspondantes, a été progressivement minée par les lobbies qui plaident pour la défense d'intérêts catégoriels, en coulisses, au sein des institutions et hors des hémicycles par des campagnes médiatiques très orchestrées. Comme le souligne Fareed Zakaria, l'homme politique américain a perdu toute liberté de parole. Constamment à l'affût des sondages d'opinion qui, plus que sa conscience ou ses convictions, orientent sa ligne de conduite, il représente les intérêts de divers groupes de pression. Les lobbies, qui emploient les meilleurs manipulateurs de symboles ${ }^{17}$, ont réduit l'ancienne aristocratie des représentants du peuple à une classe politique étroitement liée aux oligarchies politico-financières, d'autant plus indispensables dans un jeu politique où les campagnes électorales sont toujours plus onéreuses. La considération sociale dont jouissent les professionnels de la politique a considérablement baissé au cours de la seconde moitié du $\mathrm{xx}^{\mathrm{e}}$ siècle. En cela, l'Europe, tend à rejoindre l'Amérique. Le désenchantement démocratique ${ }^{18}$ caractérise les sociétés occidentales où participation électorale et engagement partisan ou civique sont partout à la baisse. Les oligarchies politico-financières ont acquis un poids considérable en maitrisant le marché des grands médias. Maurice Duverger notait qu'après 1945, les démocraties libérales avaient opéré leur mutation vers la technodémocratie, un régime qu'il voyait dominé par des vastes oligarchies réunissant acteurs publics et privés, où le gouvernement est confié à des technocrates issus de ces oligarchies ${ }^{19}$. Les partis de gouvernement des démocraties représentatives, autrefois des partis de masse bien insérés dans la société, se vident au point de ne devenir que des produits d'appel utilisés par des équipes de professionnels de la politique pour soutenir l'image et le charisme du leader qu'elles sont chargées de promouvoir auprès de l'opinion. C'est la promotion des leaders et des candidats, plutôt que le programme ou l'idéologie, qui est désormais la raison d'être des grands partis $^{20}$. Le mécanisme de la représentation politique, qui fondait les démocraties libérales, est aujourd'hui foncièrement perverti.

L'avènement d'une démocratie de masse

13 Dans la Russie post-soviétique, la dynamique représentative est presque inexistante. Elle ne s'est pas du tout développée au sein du nouveau régime. Outre la dimension césariste du pouvoir exécutif et la faiblesse du pouvoir représentatif qui en résulte, tous les observateurs notent la fragilité des partis politiques et de la société civile, peu institutionnalisée. Ainsi, le nouveau régime russe est une démocratie illibérale. Cela n'exclut pas que l'on y trouve l'élément essentiel d'une démocratie de masse: des élections, encadrées par un droit électoral et des commissions électorales, sur lesquelles règne un oligopole détenteur de toutes les ressources, constitué par les administrations (présidentielle et régionales) et les oligarchies industrielles et financières. Les élections sont un marché considérable: se faire élire coûte une petite fortune. S'y côtoient tous les professionnels de l'intermédiation politique de la 
démocratie de masse, apparus en quelques années: agences de publicité et de communication politiques, consultants et stratèges, autant de vecteurs de la capacité d'un candidat à apparaître dans les médias. De même, le lobbying, moins institutionnalisé que dans les démocraties occidentales, est une donnée fondamentale de l'économie du pouvoir. Rares sont les professionnels de la représentation politique au sens classique du terme, mais nombreux sont les députés, à Moscou comme dans les régions, qui sont les porte-voix des intérêts de groupes plus ou moins officiels. Le rôle des partis politiques est confiné à l'animation du « moment électoral ». En effet, le seul parti de masse réellement structuré à l'échelle de l'ensemble du territoire russe est le parti communiste (PCFR). Les autres partis sont des partis de cadres, parfois «jetables ", c'est-à-dire destinés à remporter une élection, ou bien des formations au service d'un leader (Iabloko, LDPR, ou le parti pro-présidentiel Russie Unie). Tous ces partis ont du mal à se structurer autour d'une idéologie et d'un programme clairement définis et relayés à tous les échelons de l'organisation. Ils semblent être condamnés à demeurer de fragiles "fédérations » du fait de leur étroite dépendance financière à l'égard de leurs « sponsors " (les cotisations des adhérents ne représentant qu'une part infime de leurs ressources). Tous ces traits sont des indices de ce que l'on pourrait appeler l'hypermodernité de la démocratie russe, un système qui, loin d'être en retard sur les démocraties occidentales, semble parfois même préfigurer leur devenir. Sans être libéral, le nouveau régime russe n'en est pas moins, si l'on s'en tient aux apparences, une démocratie de masse largement « occidentalisée ».

Le modèle de la gouvernance démocratique

Cette apparence de modernité laisse sceptiques les cercles dirigeants occidentaux, qui ne reconnaissent pas le caractère démocratique du nouveau régime russe. Les mœurs violentes et la corruption révélées par les élections ne font qu'accentuer l'étrangeté, dans tous les sens du terme, de la vie politique russe. La plupart des observateurs, en Russie comme dans les pays occidentaux, soulignent à l'envi que le nouveau régime russe est, au mieux, une démocratie Potemkine. La plupart des critiques formulées et argumentées par les chercheurs, les intellectuels ou les publicistes mettent l'accent sur la dimension proprement illibérale du nouveau régime russe. Bien des tribunes et analyses publiées dans les grands médias leur emboîtent le pas. Ainsi, la critique du nouveau régime russe est fondée sur l'existence de l'immense fossé qui le sépare du modèle de la démocratie libérale. Ce modèle étant aujourd'hui plus théorique que réellement existant, à quoi bon évaluer encore la démocratie russe par rapport à « la démocratie telle qu'elle devrait être "?

Il va de soi que la position géopolitique de la Russie influence profondément le regard porté par les dirigeants occidentaux sur son régime politique. Dans cette perspective, la critique du régime politique de la Russie d'aujourd'hui est empreinte d'une forte connotation idéologique visant à justifier, le cas échéant, l'éventuelle relégation de la Russie au rang d'ennemi. Toutefois, au-delà d'une perception guidée par des visées ou des intérêts stratégiques, il existe bien un modèle de la gouvernance démocratique, qui, même s'il n'est pas très rigoureusement défini, est devenu le seul modèle de référence opérationnel pour l'évaluation des régimes. Or, la gouvernance démocratique diffère de la démocratie représentative libérale. La notion de gouvernance démocratique (ou bonne gouvernance) part d'un constat : celui de l'atomisation de la puissance publique à l'ère de la globalisation. Il n'existe plus vraiment de gouvernement qui centralise l'autorité exécutive, mais une gouvernance qui fait intervenir plusieurs acteurs, privés et publics ${ }^{21}$, ce qui implique l'existence d'une polyarchie avec des pôles de pouvoir 
concurrents. La bonne gouvernance suppose que, dans les rapports entre ces pôles de pouvoir, les normes fixées soient respectées. La gouvernance démocratique favorise les groupes économiques et sociaux qui se constituent en groupes d'intérêt et font valoir leurs revendications dans le jeu complexe des polyarchies. Ceci fausse le jeu électoral et relègue au second plan la représentation politique des citoyens. Ce qui est vu comme relevant de la bonne gouvernance n'est plus la démocratie représentative, mais la démocratie participative ${ }^{22}$. La nouvelle démocratie participative repose implicitement sur une hiérarchie de la citoyenneté dont il faut bien admettre qu'elle relativise le poids et la signification du suffrage universel. La pleine citoyenneté suppose que le citoyen soit impliqué dans l'action collective, par le biais d'une appartenance partisane, professionnelle ou associative, c'est-à-dire par le biais d'un groupe ou d'une institution ${ }^{23}$. Se contenter de remplir son devoir civique n'est plus guère qu'une forme résiduelle de citoyenneté, un acte dont les conséquences sur l'action publique, à l'heure de la démonopolisation de la fonction exécutive, sont largement accessoires, d'où les taux d'abstention croissants. Dans les nouvelles démocraties, Amy Chua montre que la gouvernance démocratique a favorisé les groupes oligarchiques et/ou ethniques qui, au départ, étaient les plus puissants ${ }^{24}$. Aussi la gouvernance démocratique constitue-t-elle un réel obstacle à la démocratisation réelle d'un pays : meilleure légitimité du pouvoir, meilleure redistribution du revenu national, meilleure organisation de l'État, etc. La gouvernance démocratique implique l'existence d'une société civile structurée pour ne pas dégénérer en une ploutocratie ou une ethnocratie. Or, il ne faut pas perdre de vue que la montée en puissance d'une société civile structurée et de plus en plus professionnalisée, dans les pays occidentaux, pousse sur le terreau historique de la démocratie représentative.

Si les cercles dirigeants occidentaux ne considèrent pas le nouveau régime russe comme véritablement démocratique, ce n'est pas tant du fait de son caractère illibéral et de l'insuffisante représentation politique des intérêts des citoyens russes qu'en raison de l'étrangeté des mœurs politiques de la classe dirigeante russe. Quant à la population dans son ensemble, elle fait l'objet d'une grande perplexité : pourquoi cette apathie politique ? En Russie post-soviétique, le pouvoir comme la société suivent des logiques que les cercles savants et dirigeants occidentaux réprouvent ou ne comprennent pas. La connivence des élites dirigeantes, largement héritée de la configuration de l'élite du pouvoir soviétique, la nomenklatura, le nombre restreint des acteurs de la polyarchie russe et leur situation très privilégiée dans une société où l'embryon de la classe moyenne constitue en réalité une petite élite sont autant d'éléments fondamentaux de la sociologie du pouvoir post-soviétique, mais ils ne sont pas pour autant constitutifs d'une « mauvaise gouvernance ». En revanche, deux traits fondamentaux de la gouvernance russe constituent le fondement de cette perception occidentale de la gouvernance russe comme dérogatoire aux règles de la bonne gouvernance. Tout d'abord, le césarisme présidentiel affirmé, renforcé sous la présidence Poutine, est en fort décalage avec la vision excentrée et presque périphérique du pouvoir politique selon la bonne gouvernance : l'autorité exécutive est un pôle de pouvoir parmi d'autres qui, dans la mesure du possible, exerce des prérogatives qu'il définit en les négociant avec les autres pôles, placés sur un pied d'égalité. De ce point de vue, le nouveau régime russe, où la fonction régalienne du président est à la fois personnalisée et exacerbée, paraît anachronique en Europe. Ensuite, le clientélisme qui régit les rapports entre tous les acteurs politiques, économiques et sociaux, lié à une culture politique diffuse qui valorise la 
personnalisation du pouvoir au détriment des normes abstraites ${ }^{25}$ (et notamment des règles de droit), éloigne la Russie du monde occidental, qui se définit tacitement comme un espace où règnent des normes stables et impersonnelles et où les acteurs politiques ont un comportement prévisible.

La gouvernance russe : deux grands traits distinctifs

La figure présidentielle est à la fois la proue et le gouvernail du système politique. Le césarisme est l'un des fils conducteurs qu'il faut dérouler pour comprendre l'essence et le fonctionnement du nouveau régime russe. La place du chef de l'État y procède d'une conception de la légitimité qui n'est pas fondée sur la représentation du peuple souverain par le biais d'institutions (parlementaires, par exemple), mais sur la recherche d'une identité entre le peuple souverain et son dirigeant suprême. Pour reprendre cette distinction ${ }^{26}$, l'autorité des dirigeants s'y exerce le plus directement possible, en phase avec le peuple souverain, tendant à exclure les groupes, castes et corporations intermédiaires, le pouvoir présidentiel ne s'inscrit pas dans une dynamique de limitation du pouvoir d'État par le biais de la séparation des pouvoirs (conception classique de la démocratie libérale) ou dans une logique de négociation avec les divers pôles de pouvoir (bonne gouvernance) ou communion autour de la personne du président, le maximum de légitimité et de ressources pour gouverner. De ce fait, l'institution présidentielle varie en fonction de la personnalité du titulaire de la fonction. Aussi distingue-t-on trois césarismes successifs, de nature assez différente : le césarisme de transition, de Mikhaill Gorbatchev (1989-1991), le césarisme oligarchique de Boris Eltsine (1991-1999) et le césarisme bureaucratique de Vladimir Poutine (depuis 2000).

Le « système post-soviétique "

18 J'emploie à dessein le mot régime et non celui de système politique, car il s'agit de deux niveaux d'analyse. Quand intervient un changement de régime, change-t-on pour autant de système ? Comme le souligne Alain Rouquié :

Les régimes politiques peuvent apparaittre comme transitoires à l'intérieur d'un même système dont l'évolution ou la transformation obéirait à un rythme infiniment plus lent parce que mettant en jeu les forces profondes des comportements et des valeurs ${ }^{27}$.

Aussi le nouveau régime russe pourrait-il être vu comme la forme actuelle d'un métasystème politique russe dont les attributs seraient des invariants dans l'histoire et la géographie du monde russe. D'indiscutables liens de parenté unissent le nouveau régime russe et les autres régimes post-soviétiques (pays baltes exceptés). De même, on observe des similitudes entre le régime russe post-soviétique et les régimes qui se sont succédé en Russie depuis le règne d'Ivan IV le Terrible (1533-1584). L'homonymie entre césarisme et tsarisme n'est pas fortuite, puisque ces deux termes sont des synonymes ${ }^{28}$. En rompant avec les institutions soviétiques, la Russie a renoué avec la configuration du pouvoir de la fin de l'Empire. L'écrasante primauté de l'exécutif et la personnalisation du pouvoir de ses titulaires ainsi que la "hiérarchie des pouvoirs " (par opposition à la séparation des pouvoirs) en sont autant de traits distinctifs ${ }^{29}$.

Le nouveau régime russe se situe également dans la continuité immédiate du régime soviétique et, plus précisément, du régime brejnévien (1964-1983), marqué par le développement du clientélisme politique, deuxième fil directeur qu'il faut dérouler pour comprendre le nouveau régime russe. Le changement politique et social des années 1980 et 1990 a été entrepris et mis en œuvre par la génération de cadres de la 
nomenklatura qui se sont formés et ont commencé leur ascension pendant cette période où, pour la première fois depuis très longtemps dans l'histoire de l'État russe et soviétique, les responsables politiques locaux et régionaux ont la possibilité de s'enraciner au point de constituer de véritables fiefs régionaux. Des groupes de pression organisés et puissants voient le jour au sein des appareils du parti et de l'État. Ces deux types d'acteurs politiques - les dirigeants régionaux et locaux et les lobbies économiques et industriels - vont connaître un essor remarquable au cours des années 1990. Leur rôle politique, ainsi que celui des «oligarques", grands patrons issus des privatisations, est stimulé par la faiblesse de l'État. Semblant rompre avec la stratégie de Boris Eltsine qui s'appuyait alternativement sur ces divers groupes en jouant de leurs rivalités (césarisme oligarchique), Vladimir Poutine a entrepris une action visant à restaurer l'autorité de l'État en tentant de soumettre ces puissants acteurs à son autorité (césarisme bureaucratique).

D'autres régimes voient le jour après la chute de l'URSS : à l'évidence, le leadership d'un président fort caractérise tous les systèmes politiques des États de la CEI. C'est là le signe de la vivacité d'une certaine culture du pouvoir y compris là où, comme dans la Géorgie de Mikhaïl Saakachvili après décembre 2003, de nouvelles élites prétendent rompre avec Moscou et incarner un changement radical de régime. Au-delà de la gouvernance russe, il existe, dans une large mesure, une "gouvernance postsoviétique ». En plus du pluralisme électoral (Turkménistan et Biélorussie ${ }^{30}$ exceptés) et de l'omniprésence d'un pouvoir présidentiel fort, on retrouve en Ukraine ${ }^{31}$, en Géorgie, en Arménie et dans les États d'Asie centrale nombre d'institutions et de pratiques politiques comparables à celles de la Russie. Ainsi, on retrouve partout à des degrés divers et sous des formes variées, le clientélisme politique, l'opportunisme bureaucratique $^{32}$ et la corruption des fonctionnaires, la maîtrise des exécutifs sur les processus électoraux et la concentration des ressources électorales entre quelques mains, ces deux derniers traits ayant été fort bien qualifiés de "démocratie administrée " (c'est-à-dire dirigée par les exécutifs) par la sociologue Olga Krychtanovskaïa.

Une « monarchie élective » sans aristocratie

Bien entendu, la matrice de ces nouveaux régimes post-soviétiques est commune. Il faut sans doute remonter au règne de Pierre le Grand pour en comprendre toute la profondeur. Avec l'instauration de la Table des Rangs, la classe dirigeante russe est étroitement liée au pouvoir régalien par des rapports tout à la fois de hiérarchie et de dépendance. L'historien Victor Leontovitch a relevé l'absence en Russie du fondement des libertés aristocratiques qui, à l'époque moderne en Occident, sont le prélude à l'émergence des libertés démocratiques à la fin $\mathrm{du}_{\mathrm{XVIII}}^{\mathrm{e}}$ siècle $^{33}$. Jusqu'à la révolution bolchevique et davantage encore après avec la constitution de la nomenklatura, la classe dirigeante russe est une caste captive: ses membres sont privés d'un statut qui leur garantisse la stabilité de leur position et, ce faisant, des droits individuels. Appartenir à la classe dirigeante emporte des privilèges, matériels notamment, mais ne confère ni la liberté, ni la sécurité personnelle ${ }^{34}$. Qui plus est, l'absence d'un statut garanti nécessite à chaque instant l'épuisante conquête (ou la reconquête) des faveurs nécessaires au maintien au sein de la classe dirigeante. Un travail de chaque instant qui, assurément, réduisait à néant le temps nécessaire pour se cultiver et réfléchir, se consulter avec ses pairs ou poser les jalons d'une action collective. La nomenklatura va connaître une phase décisive dans les années 1960 et 1970, sous Leonid Brejnev, une phase que l'on peut aujourd'hui considérer comme un temps d'émancipation qui débouche sur la conquête, par le biais des privatisations des années 1990, d'un capital économique, soit au sens 
capitaliste classique du terme, ou en rentabilisant une position dans l'appareil administratif. Pour autant, la nomenklatura demeure jusqu'à son abolition (en 1991) une élite, sinon captive, du moins extrêmement contrainte et hiérarchisée ${ }^{35}$. Après la nomenklatura, qu'en est-il de la structure de la nouvelle classe dirigeante, de sa culture et de sa vision du monde? S'est-elle émancipée en devenant titulaire de droits de propriété $^{36}$ ? Outre que la «nouvelle » élite dirigeante n'est pas vraiment nouvelle, on constate qu'elle continue de former un groupe relativement fermé. La classe dirigeante est pourtant divisée en un nombre infini de groupes d'intérêts, mais ces derniers, très fluctuants et assez insaisissables, s'institutionnalisent peu ou pas du tout. Les conflits, liés à la maîtrise des ressources politiques, économiques ou administratives, sont éphémères et les clivages très fluctuants. C'est un facteur qui rend difficile, voire impossible, la pratique de l'alternance au pouvoir et l'institutionnalisation d'une opposition sous la forme d'un parti politique structuré et organisé.

Le pluralisme territorial

La centralité de l'institution présidentielle répond presque en écho à la géographie économique et sociale de la Fédération de Russie. Le Kremlin est au centre de la capitale, Moscou, qui est, plus que jamais, la ville-centre, le cœur politique et économique du pays, la seule métropole mondiale en interaction avec le monde extérieur, contrastant en cela avec des provinces relativement coupées des processus de globalisation, hormis quelques métropoles régionales (Saint-Pétersbourg, Ekaterinbourg ou encore Novossibirsk). Les rapports entre les grandes institutions fédérales - Président, administration présidentielle, gouvernement, Douma, Conseil de la Fédération, Banque centrale, etc. - s'inscrivent dans une topographie moscovite où se déroule l'essentiel de la vie politique de la Russie, dans les coulisses du pouvoir ou encore dans les hémicycles parlementaires ou les tribunes médiatiques, toutes situées à Moscou. Tout aspirant à la classe dirigeante, fût-ce celle d'une région, doit connaître un peu Moscou, s'y repérer dans la très mouvante géographie du pouvoir, savoir « qui est qui » au sein de l'administration présidentielle, à la Douma, dans les ministères et leurs nombreuses antichambres, qui chapeaute ou bien tire les ficelles des grandes centres économiques ou stratégiques et des très nombreux réseaux qui traversent et structurent le pouvoir en Russie. Moscou est, en soi, le lieu du pouvoir par excellence. La topographie moscovite détermine la topographie politique générale du pays.

Mais, loin s'en faut, tout ne se décide et ne se joue pas à Moscou. Le nouveau régime russe est marqué, dès sa naissance, par une tension entre le Centre et les «sujets de la Fédération ", une tension qui va déterminer bien des rapports de force politiques et économiques et imprimer profondément sa marque sur la configuration des institutions fédérales ${ }^{37}$. La Russie est bien davantage marquée par un pluralisme des territoires que par un pluralisme politique et idéologique. S'il y a eu, pendant la présidence Eltsine, une opposition politique un tant soit peu structurée, celle-ci fut constituée par les chefs des administrations régionales qui, en 1999, prirent l'initiative de mettre sur pied un "parti du pouvoir " préparant la succession de Boris Eltsine. Il s'agit de comprendre «Toutes les Russies ", au sens de l'Ancien régime, plutôt que «la Russie ». Aussi bien que le pouvoir central, et en interaction avec lui, les régimes régionaux ont subi une évolution passionnante au cours des années 1990 et 2000 . Le président Poutine a lancé sur ce terrain du fédéralisme des réformes qui posent de profondes questions : quelle est la place, à terme, des minorités nationales en Russie ? Jusqu'où peut aller l'ethnicisation du pouvoir politique dans les républiques? La Russie doit-elle être fédérale ou unitaire ? Les débats actuels sur la refonte du cadre fédéral et 
du découpage administratif du territoire ont une forte résonance dans l'histoire politique de la Russie. En effet, la recherche d'un découpage administratif adéquat et pertinent du territoire fait l'objet d'un débat presque continu entre les tenants $d u$ renforcement du centralisme et les partisans d'une forme décentralisée ou fédérale de gouvernement depuis le début du XIX ${ }^{e}$ siècle au moins. Dans ce débat entre tenants du centralisme et de l'autonomie des provinces, qui resurgit à la faveur du récent projet présidentiel de revenir sur un acquis des années 1995 - l'élection au suffrage universel (et la relative autonomie politique) des chefs des administrations régionales et des présidents des républiques - le nouveau régime russe apparait dans la plus simple et la plus complexe de ses réalités: il est d'abord et avant tout l'héritier de tous les précédents.

\section{NOTES}

1. M. Raeff, Comprendre l'ancien régime russe : État et société en Russie impériale, Paris, Seuil, $1982,249 \mathrm{p}$.

2. À l'exception majeure de l'œuvre d'A. Leroy-Beaulieu, L'Empire du Tsar et les Russes, Lausanne, L'Âge d'Homme, 1988 (1re édition Paris, 1903).

3. E. Said, L'Orientalisme : l'Orient créé par l'Occident, Paris, Seuil, 1980 (traduit et adapté de l'américain par C. Malamoud).

4. Par exemple, A. Åslund, How Russia became a market economy, Washington, The Brookings Institution, 1995.

5. Selon l'expression de Z. Brzezinski dans Le grand échiquier : l'Amérique et le reste du monde, Paris, Hachette, 1997 (traduit de l'américain par M. Bessiere et M. HerpeVoslinsky).

6. S. Cohen, Failed Crusade: America and the tragedy of Post-Communist Russia, New York, W. W. Norton, 2001.

7. B. Kagarlitsky, La Russie d'aujourd'hui : néo-libéralisme, autocratie et restauration, Paris, Parangon, 2003.

8. Le césarisme désigne un régime qui associe une « forme démocratique (l'appui populaire à un homme exceptionnel) et une réalité monarchique du pouvoir ».

M. Ganzin, Du césarisme antique au césarisme moderne, CERHIIP, Presses universitaires d'Aix-Marseille, 1999, p. 9.

9. L. Shevtsova, Putin's Russia, Washington, Carnegie Endowment for International Peace, 2003.

10. Cf. l'analyse de la notion d'illibéralisme par P. Rosanvallon, La démocratie inachevée: une histoire de la souveraineté du peuple en France, Paris, Gallimard, 2000.

11. R. Schneider, Order and progress : a political history of Brazil, Boulder, Westview Press, 1991 ; R. Levine, Father of the poor ? Vargas and his era, Cambridge University Press, 1998 12. Sur le caudillisme, les régimes autoritaires et les dictatures latino-américaines, cf. P. Vayssière, Les révolutions d'Amérique latine, Paris, Seuil, 2001 (1re édition 1991),

A. Rouquié, L'État militaire en Amérique latine, Paris, Seuil, 1982. 
13. La démocratie est aujourd'hui un instrument défini par les politiques publiques internationales des États-Unis et de l'UE. En ce qui concerne les États-Unis, le type de démocratie préconisé pour la Russie est assez bien décrit dans le Russian Democracy Actvoté, dans sa dernière version, par le Congrès des États-Unis en octobre 2002.

14. F. Zakaria, L'avenir de la liberté. La démocratie illibérale aux États-Unis et dans le monde, Paris, Odile Jacob, 2003 (1re édition en américain, New York, 2003), dans la traduction de D. Roche.

15. C. Schmitt, Parlementarisme et démocratie, Paris, Seuil, 1988 (1re édition en allemand, Berlin, 1923), dans la traduction de J.-L. Schlegel, p. 47.

16. Au sens où $\mathrm{M}$. Weber entend le métier d'homme politique, animé par une vocation. Cf. «Le métier et la vocation d'homme politique ", in $\mathrm{M}$. Weber, Le savant et le politique, Paris, Plon, 1959 (édition originale en allemand, 1919).

17. R. Reich, L'économie mondialisée, Paris, Dunod, 1997 (traduit de l'américain par

D. Ternam).

18. Pour une synthèse de cette crise de la représentation dans une démocratie représentative établie comme la France, $c f$. P. Perrineau, Désenchantement démocratique, La Tour d'Aigues, Éditions de l'Aube, 2003.

19. M. Duverger, Janus : les deux faces de l'Occident, Paris, Fayard, 1972.

20. Forza Italia est à ce titre emblématique. Cf. P. Musso, Berlusconi, le nouveau Prince, La Tour d'Aigues, Éditions de l'Aube, 2003.

21. R. Balme, D. Chabanet, « Action collective et gouvernance dans l'Union européenne ", in

R. Balme et al. (dir.), L'action collective en Europe, Paris, Presses de Science Po, 2002, p. 108-109.

22. G. Hermet, « Un régime à pluralisme limité : à propos de la gouvernance démocratique ", Revue française de science politique, vol. 54, n 1, 2004, p. 159-178.

23. Ainsi, en français, « citoyen » est devenu un adjectif associé à des actes, des attitudes et des comportements, et n'est plus seulement un substantif. Ce nouveau sens implique que la citoyenneté suppose aujourd'hui d'agir dans l'espace public, et non plus se contenter de cet acte par excellence anonyme et intime qu'est le vote.

24. A. Chua, World on Fire: How Exporting Free Market Democracy Breeds Ethnic Hatred and Global Instability, New York, Doubleday Books, 2002.

25. Cf. l'ouvrage de référence de M. Afanas'ev, Klientelizm i rossijskaja gosudarstvennost' [Le clientélisme et l'État russe], Moskva, MONF, 2000.

26. C. Schmitt, Parlementarisme et démocratie, op. cit., 1988.

27. A. Rouquié, «Changement politique et transformation des régimes ", in M. Grawitz, J. Leca, Traité de science politique, vol. 2, Paris, Puf, 1985, p. 601.

28. Le russe carizm (tsarisme) est un dérivé de cesarizm (césarisme).

29. Pour une analyse très éclairante de la nature et du fonctionnement de l'autocratie russe à son crépuscule, N. A. Zakharov, Russkaja politiceskaja sistema [Le système politique russe], Novocerkassk, 1912, rééd. Moscou, 2002.

30. A. Goujon, « Le "loukachisme" ou le populisme autoritaire en Biélorussie », Politique et sociétés, vol. 21, $\mathrm{n}^{\circ} 2,2002$, p. 29-50.

31. P. Kubicek, Unbroken Ties : the State, Interest Associations and Corporatism in Post-Soviet Ukraine, Ann Arbor, University of Michigan Press, 2000.

32. G. Favarel-Garrigues et $\mathrm{K}$. Rousselet, La société russe en quête d'ordre : avec Vladimir Poutine? Paris, Autrement, 2004.

33. V. Leontovitch, Histoire du libéralisme en Russie, Paris, Fayard, 1979 (traduit de l'allemand par O. Hansen-Loeve), éd. originale à Francfort-sur-le-Main, 1957. 
34. Aujourd'hui, l'insécurité est le sentiment dominant au sein de la classe dirigeante post-soviétique, la peur des élites pour leur sécurité personnelle étant très bien soulignée dans tous les travaux récents de V. Shlapentokh.

35. M. Voslensky, La nomenklatura. Les privilégiés en URSS, Paris, Belfond, 1980.

36. C. Freeland, Sale of the century : the inside story of the second Russian Revolution, Londres, Little and Brown, 2000.

37. M. Mendras (dir.), « Russie :le gouvernement des provinces », Nouveaux Mondes, Genève, CRES, n 7, 1997.

\section{RÉSUMÉS}

Jean-Robert Raviot s'attache à inventorier et à décrire pour la Russie en recourant à une terminologie différenciée, révélatrice des mutations en cours et des points de vue adoptés: hyper-modernité démocratique, démocratie dirigée et illibérale, gouvernance démocratique, technodémocratie, démocratie de masse, fédération de d'États, etc. Ces nouvelles formes semblent s'inscrire dans des continuités historiques fortes dans le cas russe en particulier: le césarisme tel qu'il apparaît aujourd'hui en Russie sous diverses formes, césarisme de transition, oligarchique ou bureaucratique, relie celle-ci à son histoire longue tout en l'ancrant dans une modernité "post-moderne ». Loin de faire de la Russie une exception atypique dans le monde politique contemporain dont nombre de poncifs occidentaux l'accablent, l'auteur resitue cette dernière dans la lignée des régimes autoritaires tels qu'on peut les trouver en Amérique latine ou ailleurs et donnent des clés de lecture des autres régimes post-soviétiques.

Jean Robert Raviot. Understanding the new Russian political regime.

The author is describing the Russian political system through the use of a new terminology, bringing to light occurring mutations: democratic hyper-modernity, directed and illiberal democracy, democratic governance, techno-democracy, mass democracy etc. For him, these new forms are to be placed in a strong historic continuity: "cesarism" as it appears in Russia today under a variety of forms - "transition cesarism", "oligarchic or bureaucratic cesarism." It links it back to Russia's long history whilst anchoring it in a "post-modern" modernity. Far from making Russia an atypical example in the contemporary political world, a cliché repeated by so many western analysts, the author places it back in the line of authoritarian regimes such as can be found in Latin America or elsewhere, and provides useful clues for understanding other postsoviet regimes.

\section{AUTEUR}

\section{JEAN-ROBERT RAVIOT}

Maître de conférences, département d'études slaves, université Paris X-Nanterre, jrraviot@wanadoo.fr 